\title{
ANALYSIS OF THE DESCRIPTOR ROESSER MODEL WITH THE USE OF THE DRAZIN INVERSE
}

\author{
TADEUSZ KACZOREK ${ }^{a}$ \\ ${ }^{a}$ Faculty of Electrical Engineering \\ Białystok University of Technology, ul. Wiejska 45D, 15-351 Białystok, Poland \\ e-mail: kaczorekeisep.pw.edu.pl
}

\begin{abstract}
A method of analysis for a class of descriptor 2D discrete-time linear systems described by the Roesser model with a regular pencil is proposed. The method is based on the transformation of the model to a special form with the use of elementary row and column operations and on the application of a Drazin inverse of matrices to handle the model. The method is illustrated with a numerical example.
\end{abstract}

Keywords: Drazin inverse, descriptor, Roesser model, descriptor-time, 2D linear system.

\section{Introduction}

Descriptor (singular) linear systems were considered in many papers and books (Bru et al., 2003; 2000; 2002; Campbell et al., 1976; Dai, 1989; Dodig and Stosic, 2009; Fahmy and O'Reill, 1989; Gantmecher, 1960; Duan, 2010; Kaczorek, 2014a; 2011a; 2004; 2013; 2011b; 2011c; 2011d; 1992; Kucera and Zagalak, 1988; Van Dooren, 1979). The eigenvalues and invariants assignment by state and output feedbacks was investigated by Fahmy and O'Reill (1989) as well as Kaczorek (2004; 1992), who also discussed the minimum energy control of descriptor linear systems (Kaczorek, 2014b; 2014c). Computation of Kronecker's canonical form of the singular pencil was analyzed by Van Dooren (1979), while positive linear systems with different fractional orders were addressed by Kaczorek (2010), along with selected problems in the theory of fractional linear systems (Kaczorek, 2011d).

Descriptor standard positive linear systems were addressed with the use of the Drazin inverse by Bru et al. (2003; 2000; 2002), Campbell et al. (1976), and Kaczorek (2014a; 2011d; 1992), who also applied the shuffle algorithm to check the positivity of descriptor linear systems (Kaczorek, 2011a). The stability of positive descriptor systems was investigated by Virnik (2008), while reduction and decomposition of descriptor fractional discrete-time linear systems were considered by Kaczorek (2011b), who also introduced a new class of descriptor fractional linear discrete-time systems
(Kaczorek, 2011c).

The Drazin inverse for finding the solution to the state equation of fractional continuous-time linear systems was applied by Kaczorek (2014a), and the controllability, reachability and minimum energy control of fractional discrete-time linear systems with delays in state were investigated by Busłowicz (2014).

In this paper, a Drazin inverse of matrices will be used in the analysis of descriptor discrete-time 2D linear systems regular pencils described by the Roesser model.

The paper is organized as follows. In Section 2, basic definitions and theorems concerning descriptor discrete-time linear systems with regular pencils are presented. The problem of the analysis of descriptor systems described by the Roesser model is formulated and solved in Section 3. The proposed method is illustrated with a numerical example in Section 4. Concluding remarks are given in Section 5.

The following notation will be used: $\mathbb{R}$, the set of real numbers; $\mathbb{R}^{n \times m}$, the set of $n \times m$ real matrices and $\mathbb{R}^{n}=$ $\mathbb{R}^{n \times 1} ; \mathbb{Z}_{+}$, the set of nonnegative integers; $I_{n}$, the $n \times n$ identity matrix; ker $A$ (im $A$ ), the kernel (image) of the matrix.

\section{Descriptor discrete-time linear systems}

Consider the descriptor discrete-time linear system

$$
E x_{i+1}=A x_{i}+B u_{i}, \quad i \in \mathbb{Z}_{+},
$$


where $x_{i} \in \mathbb{R}^{n}$ is the state vector, $u_{i} \in \mathbb{R}^{m}$ is the input vector, $E, A \in \mathbb{R}^{n \times n}, B \in \mathbb{R}^{n \times m}$.

It is assumed that $\operatorname{det} E=0$, but

$$
\operatorname{det}[E s-A] \neq 0 \quad \text { for some } c \in \mathbb{C} .
$$

Assuming that, for some chosen $c \in \mathbb{C}, \operatorname{det}[E c-A] \neq 0$ and premultiplying (1) by $[E c-A]^{-1}$, we obtain

$$
\bar{E} x_{i+1}=\bar{A} x_{i}+\bar{B} u_{i},
$$

where

$$
\begin{aligned}
\bar{E} & =[E c-A]^{-1} E, \\
\bar{A} & =[E c-A]^{-1} A, \\
\bar{B} & =[E c-A]^{-1} B .
\end{aligned}
$$

Note that Eqns. (1) and (3a) have the same solution $x_{i}$, $i \in \mathbb{Z}_{+}$.

Definition 1. (Campbell et al., 1976; Kaczorek, 1992) The smallest nonnegative integer $q$ is called the index of the matrix $\bar{E} \in \mathbb{R}^{n \times n}$ if

$$
\operatorname{rank} \bar{E}^{q}=\operatorname{rank} \bar{E}^{q+1} \text {. }
$$

Definition 2. (Campbell et al., 1976; Kaczorek, 1992) A matrix $\bar{E}^{D}$ is called the Drazin inverse of $\bar{E} \in \mathbb{R}^{n \times n}$ if it satisfies the conditions

$$
\begin{aligned}
\bar{E} \bar{E}^{D} & =\bar{E}^{D} \bar{E}, \\
\bar{E}^{D} \bar{E} \bar{E}^{D} & =\bar{E}^{D}, \\
\bar{E}^{D} \bar{E}^{q+1} & =\bar{E}^{q},
\end{aligned}
$$

where $q$ is the index of $\bar{E}$ defined by (4).

The Drazin inverse $\bar{E}^{D}$ of a square matrix $\bar{E}$ always exists and is unique (Campbell et al., 1976; Kaczorek, 1992). If det $\bar{E} \neq 0$, then $\bar{E}^{D}=\bar{E}^{-1}$. Some methods for computation of the Drazin inverse are given by Kaczorek (1992) and Van Dooren (1979), and are summarized in Appendix.

Theorem 1. (Kaczorek, 1992) The matrices $\bar{E}$ and $\bar{A}$ defined by (3b) satisfy the following equalities:

$$
\begin{gathered}
\bar{A} \bar{E}=\bar{E} \bar{A}, \quad \bar{A}^{D} \bar{E}=\bar{E} \bar{A}^{D}, \\
\bar{E}^{D} \bar{A}=\bar{A} \bar{E}^{D}, \quad \bar{A}^{D} \bar{E}^{D}=\bar{E}^{D} \bar{A}^{D}, \\
\quad \operatorname{ker} \bar{A} \cap \operatorname{ker} \bar{E}=\{0\}, \\
\bar{E}=T\left[\begin{array}{cc}
J & 0 \\
0 & N
\end{array}\right] T^{-1}, \\
\bar{E}^{D}=T\left[\begin{array}{cc}
J^{-1} & 0 \\
0 & 0
\end{array}\right] T^{-1}, \\
\left(I_{n}-\bar{E} \bar{E}^{D}\right) \bar{A} \bar{A}^{D}=I_{n}-\bar{E} \bar{E}^{D}, \\
\left(I_{n}-\bar{E} \bar{E}^{D}\right)\left(\bar{E} \bar{A}^{D}\right)^{q}=0,
\end{gathered}
$$

$\operatorname{det} T \neq 0, J \in \mathbb{R}^{n_{1} \times n_{1}}$, is nonsingular, $N \in \mathbb{R}^{n_{2} \times n_{2}}$ is nilpotent, $n_{1}+n_{2}=n$.
Theorem 2. (Campbell et al., 1976; Kaczorek, 1992) The solution of Eqn. (3) is given by

$$
\begin{aligned}
x_{i}= & \left(\bar{E}^{D} \bar{A}\right)^{i} \bar{E}^{D} \bar{E} v+\sum_{k=0}^{i-1} \bar{E}^{D}\left(\bar{E}^{D} \bar{A}\right)^{i-k-1} \bar{B} u_{k} \\
& +\left(\bar{E} \bar{E}^{D}-I_{n}\right) \sum_{k=0}^{q-1}\left(\bar{E} \bar{A}^{D}\right)^{k} \bar{A}^{D} \bar{B} u_{i+k},
\end{aligned}
$$

where $v \in \mathbb{R}^{n}$ is arbitrary and $q$ is the index of $E$.

From (7), for $i=0$ we have

$$
x_{0}=\bar{E}^{D} \bar{E} v+\left(\bar{E} \bar{E}^{D}-I_{n}\right) \sum_{k=0}^{q-1}\left(\bar{E} \bar{A}^{D}\right)^{k} \bar{A}^{D} \bar{B} u_{k} .
$$

Therefore, for given admissible $u_{i}$, the consistent initial conditions should satisfy the equality (8). In a particular case for $u_{i}=0$ we have $x_{0}=\bar{E}^{D} \bar{E} v$ and $x_{0} \in$ $\operatorname{Im}\left(\bar{E}^{D} \bar{E}\right)$, where $\operatorname{Im}$ denotes the image of $\bar{E}^{D} \bar{E}$.

Theorem 3. Let

$$
\begin{aligned}
& P=\bar{E} \bar{E}^{D}, \\
& Q=\bar{E}^{D} \bar{A} .
\end{aligned}
$$

Then

(i)

$$
P^{k}=P \quad \text { for } k=2,3, \ldots ;
$$

(ii)

$$
P Q=Q P=Q
$$

(iii)

$$
P \bar{E}^{D}=\bar{E}^{D} P=\bar{E}^{D} ;
$$

(iv) if there exists a vector $v \in \mathbb{R}^{n}$ such that

$$
v^{T} \bar{E}=0
$$

then

$$
v^{T} \bar{E}^{D}=0
$$

Proof. Using (9), we obtain

$$
P^{2}=\bar{E} \bar{E}^{D} \bar{E} \bar{E}^{D}=\bar{E} \bar{E}^{D}=P
$$

since, by (5b), $\bar{E}^{D} \bar{E} \bar{E}^{D}=\bar{E}^{D}$ and, by induction,

$$
P^{k}=P^{k-1} P=\bar{E} \bar{E}^{D} \bar{E} \bar{E}^{D}=P^{2}=P
$$

for $k=2,3, \ldots$.

Using (9) and (10), we obtain

$$
P Q=\bar{E} \bar{E}^{D} \bar{E}^{D} \bar{A}=\bar{E}^{D} \bar{E} \bar{E}^{D} \bar{A}=\bar{E}^{D} \bar{A}=Q
$$

and

$$
\begin{aligned}
Q P & =\bar{E}^{D} \bar{A} \bar{E} \bar{E}^{D}=\bar{E}^{D} \bar{E} \bar{A} \bar{E}^{D} \\
& =\bar{E}^{D} \bar{E} \bar{E}^{D} \bar{A}=\bar{E}^{D} \bar{A}=Q .
\end{aligned}
$$


Using (9), (5a) and (5b), we obtain

$$
P \bar{E}^{D}=\bar{E} \bar{E}^{D} \bar{E}^{D}=\bar{E}^{D} \bar{E} \bar{E}^{D}=\bar{E}^{D}
$$

and

$$
\bar{E}^{D} P=\bar{E}^{D} \bar{E} \bar{E}^{D}=\bar{E}^{D} .
$$

Taking into account that $\bar{E}=V W$ (see Appendix) and

$$
\bar{E}^{D}=V[W \bar{E} V]^{-1} W
$$

we obtain

$$
v^{T} \bar{E}^{D}=v^{T} V[W \bar{E} V]^{-1} W=0
$$

since $v^{T} V=0$

The following elementary row (resp. column) operations will be used:

1. Multiplication of the $i$-th row (resp. column) by a real number $c$. This operation will be denoted by $L[i \times c]$ (resp. $R[i \times c]$ ).

2. Addition to the $i$-th row (resp. column) of the $j$-th row (resp. column) multiplied by a real number $c$. This operation will be denoted by $L[i+j \times c]$ (resp. $R[i+j \times c])$

3. Interchange of the $i$-th and $j$-th rows (columns). This operation will be denoted by $L[i, j]$ (resp. $R[i, j]$ ).

\section{Problem formulation and solution}

Consider the descriptor Roesser model

$$
E\left[\begin{array}{l}
x_{i+1, j}^{h} \\
x_{i, j+1}^{v}
\end{array}\right]=A\left[\begin{array}{c}
x_{i, j}^{h} \\
x_{i, j}^{v}
\end{array}\right]+B u_{i, j}
$$

where $x_{i, j}^{h} \in \mathbb{R}^{n_{1}}, x_{i, j}^{v} \in \mathbb{R}^{n_{2}}$ are the horizontal and vertical state vectors $u_{i, j} \in \mathbb{R}^{m}$ is the input vector and $E, A \in \mathbb{R}^{n \times n}, B \in \mathbb{R}^{n \times m}, n=n_{1}+n_{2}$.

It is assumed that det $E=0$, but

$$
\begin{aligned}
\operatorname{det}\left[E\left[\begin{array}{cc}
I_{n_{1}} z_{1} & 0 \\
0 & I_{n_{2}} z_{2}
\end{array}\right]\right. & -A] \neq 0 \\
& \text { for some } z_{1}, z_{2} \in \mathbb{C} .
\end{aligned}
$$

It is also assumed that, premultiplying (24) by a matrix $P \in \mathbb{R}^{n \times n}$ of the elementary row operations and introducing the new state vector

$$
\begin{aligned}
{\left[\begin{array}{c}
\bar{x}_{i+1, j}^{h} \\
\bar{x}_{i, j+1}^{v}
\end{array}\right]=Q\left[\begin{array}{c}
x_{i, j}^{h} \\
x_{i, j}^{v}
\end{array}\right], } & \\
Q & \in \mathbb{R}^{n \times n}, \quad \operatorname{det} Q \neq 0,
\end{aligned}
$$

Eqn. (24) can be written in the following form:
Case 1:

$$
\begin{aligned}
& {\left[\begin{array}{cc}
E_{h} & 0 \\
0 & E_{v}
\end{array}\right]\left[\begin{array}{l}
\bar{x}_{i+1, j}^{h} \\
\bar{x}_{i, j+1}^{v}
\end{array}\right]} \\
& =\left[\begin{array}{cc}
A_{11} & 0 \\
A_{21} & A_{22}
\end{array}\right]\left[\begin{array}{l}
\bar{x}_{i, j}^{h} \\
\bar{x}_{i, j}^{v}
\end{array}\right]+\left[\begin{array}{l}
B_{1} \\
B_{2}
\end{array}\right] u_{i, j} .
\end{aligned}
$$

Case 2:

$$
\begin{aligned}
& {\left[\begin{array}{cc}
E_{h} & 0 \\
0 & E_{v}
\end{array}\right]\left[\begin{array}{l}
\bar{x}_{i+1, j}^{h} \\
\bar{x}_{i, j+1}^{v}
\end{array}\right]} \\
& =\left[\begin{array}{cc}
A_{11} & A_{12} \\
0 & A_{22}
\end{array}\right]\left[\begin{array}{l}
\bar{x}_{i, j}^{h} \\
\bar{x}_{i, j}^{v}
\end{array}\right]+\left[\begin{array}{l}
B_{1} \\
B_{2}
\end{array}\right] u_{i, j},
\end{aligned}
$$

where

$$
\left[\begin{array}{cc}
E_{h} & 0 \\
0 & E_{v}
\end{array}\right]=P E Q^{-1}, \quad E_{h} \in \mathbb{R}^{n_{1} \times n_{1}},
$$$$
E_{v} \in \mathbb{R}^{n_{2} \times n_{2}}, \quad \operatorname{det} E_{h}=0, \quad \operatorname{det} E_{v}=0
$$

$\left[\begin{array}{cc}A_{11} & 0 \\ A_{21} & A_{22}\end{array}\right]=P A Q^{-1}, \quad A_{11} \in \mathbb{R}^{n_{1} \times n_{1}}$,

$A_{21} \in \mathbb{R}^{n_{2} \times n_{1}}, A_{22} \in \mathbb{R}^{n_{2} \times n_{2}}$,

$\left[\begin{array}{cc}A_{11} & A_{12} \\ 0 & A_{22}\end{array}\right]=P A Q^{-1}, A_{11} \in \mathbb{R}^{n_{1} \times n_{1}}$

$A_{12} \in \mathbb{R}^{n_{1} \times n_{2}}, \quad A_{22} \in \mathbb{R}^{n_{2} \times n_{2}}$,

$\left[\begin{array}{c}B_{1} \\ B_{2}\end{array}\right]=P B, \quad B_{1} \in \mathbb{R}^{n_{1} \times m}, B_{2} \in \mathbb{R}^{n_{2} \times m}$.

In Case 1, from (27a) we have

$$
\begin{aligned}
& E_{h} \bar{x}_{i+1, j}^{h}=A_{11} \bar{x}_{i, j}^{h}+B_{1} u_{i, j}, \\
& E_{v} \bar{x}_{i, j+1}^{v}=A_{21} \bar{x}_{i, j}^{h}+A_{22} \bar{x}_{i, j}^{v}+B_{2} u_{i, j},
\end{aligned}
$$

and, in Case 2, from (27b) we have

$$
\begin{aligned}
& E_{h} \bar{x}_{i+1, j}^{h}=A_{11} \bar{x}_{i, j}^{h}+A_{12} \bar{x}_{i, j}^{v}+B_{1} u_{i, j}, \\
& E_{v} \bar{x}_{i, j+1}^{v}=A_{22} \bar{x}_{i, j}^{v}+B_{2} u_{i, j} .
\end{aligned}
$$

From the assumption (25) for Case 1 it follows that

$$
\operatorname{det}\left[E_{h} z_{1}-A_{11}\right] \neq 0 \quad \text { for some } z_{1} \in \mathbb{C} \text {. }
$$

Therefore, there exists a number $c_{1} \in \mathbb{C}$ such that $\operatorname{det}\left[E_{h} c_{1}-A_{11}\right] \neq 0$ and, premultiplying (27a) by $\left[E_{h} c_{1}-A_{11}\right]^{-1}$, we obtain

$$
\bar{E}_{h} \bar{x}_{i+1, j}^{h}=\bar{A}_{11} \bar{x}_{i, j}^{h}+\bar{B}_{1} u_{i, j},
$$

where

$$
\begin{aligned}
\bar{E}_{h} & =\left[E_{h} c_{1}-A_{11}\right]^{-1} E_{h}, \\
\bar{A}_{11} & =\left[E_{h} c_{1}-A_{11}\right]^{-1} A_{11}, \\
\bar{B}_{1} & =\left[E_{h} c_{1}-A_{11}\right]^{-1} B_{1} .
\end{aligned}
$$

Let $\bar{E}_{h}^{D}\left(\bar{A}_{11}^{D}\right)$ be the Drazin inverse of the matrix $\bar{E}_{h}$ 
$\left(\bar{A}_{11}\right)$. Then, from Theorem 2 , we have

$$
\begin{aligned}
\bar{x}_{i, j}^{h}= & \left(\bar{E}_{h}^{D} \bar{A}_{11}\right)^{i} \bar{E}_{h}^{D} \bar{E}_{h} v_{1} \\
& +\sum_{k=0}^{i-1} \bar{E}_{h}^{D}\left(\bar{E}_{h}^{D} \bar{A}_{11}\right)^{i-k-1} \bar{B}_{1} u_{k, j} \\
& +\left(\bar{E}_{h} \bar{E}_{h}^{D}-I_{n_{1}}\right) \sum_{k=0}^{q_{1}-1}\left(\bar{E}_{h} \bar{A}_{11}^{D}\right)^{k} \bar{A}_{11}^{D} \bar{B}_{1} u_{i+k, j},
\end{aligned}
$$

where $q_{1}$ is the index of $\bar{E}_{h}$ and $v_{1} \in \mathbb{R}^{n_{1}}$ is arbitrary depending on $j$.

Substituting (32) into (27b) yields

$$
E_{v} \bar{x}_{i, j+1}^{v}=A_{22} \bar{x}_{i, j}^{v}+\hat{u}_{i, j}+\hat{w}_{i},
$$

where

$$
\begin{aligned}
\hat{u}_{i, j}= & \sum_{k=0}^{i-1} A_{21} \bar{E}_{h}^{D}\left(\bar{E}_{h}^{D} \bar{A}_{11}\right)^{i-k-1} \bar{B}_{1} u_{k, j} \\
& +A_{21}\left(\bar{E}_{h} \bar{E}_{h}^{D}-I_{n_{1}}\right) \\
& \times \sum_{k=0}^{q_{1}-1}\left(\bar{E}_{h} \bar{A}_{11}^{D}\right)^{k} \bar{A}_{11}^{D} \bar{B}_{1} u_{i+k, j}+B_{2} u_{i, j} \\
\hat{w}_{i}= & A_{21}\left(\bar{E}_{h}^{D} \bar{A}_{11}\right)^{i} \bar{E}_{h}^{D} \bar{E}_{h} v_{1} .
\end{aligned}
$$

From the assumption (25) it follows that

$$
\operatorname{det}\left[E_{v} z_{2}-A_{22}\right] \neq 0 \quad \text { for some } z_{2} \in \mathbb{C} \text {. }
$$

Therefore, there exists a number $c_{2} \in \mathbb{C}$ such that $\operatorname{det}\left[E_{v} c_{2}-A_{22}\right] \neq 0$ and, premultiplying (33) by $\left[E_{v} c_{2}-\right.$ $\left.A_{22}\right]^{-1}$, we obtain

$$
\bar{E}_{v} \bar{x}_{i, j+1}^{v}=\bar{A}_{22} \bar{x}_{i, j}^{v}+\bar{B}_{2}\left(\hat{u}_{i, j}+\hat{w}_{i}\right),
$$

where

$$
\begin{aligned}
\bar{E}_{v} & =\left[E_{v} c_{2}-A_{22}\right]^{-1} E_{v}, \\
\bar{A}_{22} & =\left[E_{v} c_{2}-A_{22}\right]^{-1} A_{22}, \\
\bar{B}_{2} & =\left[E_{v} c_{2}-A_{22}\right]^{-1} .
\end{aligned}
$$

Let $\bar{E}_{v}^{D}\left(\bar{A}_{22}^{D}\right)$ be the Drazin inverse of the matrix $\bar{E}_{v}$ $\left(\bar{A}_{22}\right)$. Then, from Theorem 2, we have

$$
\begin{aligned}
\bar{x}_{i, j}^{v}= & \left(\bar{E}_{v}^{D} \bar{A}_{22}\right)^{j} \bar{E}_{v}^{D} \bar{E}_{v} v_{2} \\
& +\sum_{l=0}^{j-1} \bar{E}_{v}^{D}\left(\bar{E}_{v}^{D} \bar{A}_{22}\right)^{j-l-1} \bar{B}_{2}\left(\hat{u}_{i, l}+\hat{w}_{i}\right) \\
& +\left(\bar{E}_{v} \bar{E}_{v}^{D}-I_{n_{2}}\right) \sum_{l=0}^{q_{2}-1}\left(\bar{E}_{v} \bar{A}_{22}^{D}\right)^{l} \\
& \times \bar{A}_{22}^{D} \bar{B}_{2}\left(\hat{u}_{i, j+l}+\hat{w}_{i}\right),
\end{aligned}
$$

where $q_{2}$ is the index of $\bar{E}_{v}$ and $v_{2} \in \mathbb{R}^{n_{2}}$ is arbitrary depending on $i$.
Knowing $\bar{x}_{i, j}^{h}$ and $\bar{x}_{i, j}^{v}$ we can find the solution of Eqn. (27) from (26) and obtain

$$
\left[\begin{array}{c}
x_{i, j}^{h} \\
x_{i, j}^{v}
\end{array}\right]=Q^{-1}\left[\begin{array}{c}
\bar{x}_{i, j}^{h} \\
\bar{x}_{i, j}^{v}
\end{array}\right] .
$$

Therefore, the following result has been proved.

Theorem 4. The solution of Eqn. (27a) is given by (37) and the vectors $\bar{x}_{i, j}^{h}$ and $\bar{x}_{i, j}^{v}$ are defined by (32) and (36), respectively.

From the assumption (25), for Case 2 it follows that

$$
\operatorname{det}\left[E_{v} z_{2}-A_{22}\right] \neq 0 \quad \text { for some } z_{2} \in \mathbb{C} .
$$

Therefore, there exists a number $c_{2} \in \mathbb{C}$ such that $\operatorname{det}\left[E_{v} c_{2}-A_{22}\right] \neq 0$ and, premultiplying (27b) by $\left[E_{v} c_{2}-A_{22}\right]^{-1}$, we obtain

$$
\bar{E}_{v} \bar{x}_{i, j+1}^{v}=\bar{A}_{22} \bar{x}_{i, j}^{v}+\bar{B}_{2} u_{i, j},
$$

where

$$
\begin{aligned}
\bar{E}_{v} & =\left[E_{v} c_{2}-A_{22}\right]^{-1} E_{v}, \\
\bar{A}_{22} & =\left[E_{v} c_{2}-A_{22}\right]^{-1} A_{22}, \\
\bar{B}_{2} & =\left[E_{v} c_{2}-A_{22}\right]^{-1} B_{2} .
\end{aligned}
$$

Let $\bar{E}_{v}^{D}\left(\bar{A}_{22}^{D}\right)$ be the Drazin inverse of the matrix $\bar{E}_{v}$ $\left(\bar{A}_{22}\right)$. Then, from Theorem 2 , we have

$$
\begin{aligned}
\bar{x}_{i, j}^{v}= & \left(\bar{E}_{v}^{D} \bar{A}_{22}\right)^{j} \bar{E}_{v}^{D} \bar{E}_{v} v_{3} \\
& +\sum_{l=0}^{j-1} \bar{E}_{v}^{D}\left(\bar{E}_{v}^{D} \bar{A}_{22}\right)^{j-l-1} \bar{B}_{2} u_{i, l} \\
& +\left(\bar{E}_{v} \bar{E}_{v}^{D}-I_{n_{2}}\right) \sum_{l=0}^{q_{2}-1}\left(\bar{E}_{v} \bar{A}_{22}^{D}\right)^{l} \bar{A}_{22}^{D} \bar{B}_{2} u_{i, j+l}
\end{aligned}
$$

where $q_{2}$ is the index of $\bar{E}_{v}$ and $v_{3} \in \mathbb{R}^{n_{2}}$ is arbitrary.

Substituting (40) into (27a) yields

$$
E_{h} \bar{x}_{i+1, j}^{h}=A_{11} \bar{x}_{i, j}^{h}+\tilde{u}_{i, j}+\tilde{w}_{j},
$$

where

$$
\begin{aligned}
\tilde{u}_{i, j}= & \sum_{l=0}^{j-1} A_{21} \bar{E}_{v}^{D}\left(\bar{E}_{v}^{D} \bar{A}_{22}\right)^{j-l-1} \bar{B}_{2} u_{i, l} \\
& +A_{12}\left(\bar{E}_{v} \bar{E}_{v}^{D}-I_{n_{2}}\right) \sum_{l=0}^{q_{2}-1}\left(\bar{E}_{v} \bar{A}_{22}^{D}\right)^{l} \\
& \times \bar{A}_{22}^{D} \bar{B}_{2} u_{i, j+l}+B_{1} u_{i, j} \\
\tilde{w}_{j}= & A_{12}\left(\bar{E}_{v}^{D} \bar{A}_{22}\right)^{j} \bar{E}_{v}^{D} \bar{E}_{v} v_{3}
\end{aligned}
$$

From the assumption (25) it follows that

$$
\operatorname{det}\left[E_{h} z_{1}-A_{11}\right] \neq 0 \quad \text { for some } z_{1} \in \mathbb{C} \text {. }
$$


Therefore, there exists a number $c_{1} \in \mathbb{C}$ such that $\operatorname{det}\left[E_{h} c_{1}-A_{11}\right] \neq 0$ and, premultiplying (41a) by $\left[E_{h} c_{1}-A_{11}\right]^{-1}$, we obtain

$$
\tilde{E}_{h} \bar{x}_{i+1, j}^{h}=\tilde{A}_{11} \bar{x}_{i, j}^{h}+\tilde{B}_{1}\left(\tilde{u}_{i, j}+\tilde{w}_{j}\right)
$$

where

$$
\begin{aligned}
\tilde{E}_{h} & =\left[E_{h} c_{1}-A_{11}\right]^{-1} E_{h}, \\
\tilde{A}_{11} & =\left[E_{h} c_{1}-A_{11}\right]^{-1} A_{11}, \\
\tilde{B}_{1} & =\left[E_{h} c_{1}-A_{11}\right]^{-1} .
\end{aligned}
$$

Let $\tilde{E}_{h}^{D}\left(\tilde{A}_{11}^{D}\right)$ be the Drazin inverse of the matrix $\tilde{E}_{h}$ $\left(\tilde{A}_{11}\right)$. Then, from Theorem 2, we have

$$
\begin{aligned}
\bar{x}_{i, j}^{h}= & \left(\tilde{E}_{h}^{D} \tilde{A}_{11}\right)^{i} \tilde{E}_{h}^{D} \tilde{E}_{h} v_{4} \\
& +\sum_{k=0}^{i-1} \tilde{E}_{h}^{D}\left(\tilde{E}_{h}^{D} \tilde{A}_{11}\right)^{i-k-1} \tilde{B}_{1}\left(\tilde{u}_{k, j}+\tilde{w}_{j}\right) \\
& +\left(\tilde{E}_{h} \tilde{E}_{h}^{D}-I_{n_{1}}\right) \sum_{k=0}^{q_{1}-1}\left(\tilde{E}_{h} \tilde{A}_{11}^{D}\right)^{k} \\
& \times \tilde{A}_{11}^{D} \tilde{B}_{1}\left(\tilde{u}_{i+k, j}+\tilde{w}_{j}\right)
\end{aligned}
$$

where $q_{1}$ is the index of $\tilde{E}_{h}$ and $v_{4} \in \mathbb{R}^{n_{1}}$ is arbitrary.

Knowing $\bar{x}_{i, j}^{h}$ and $\bar{x}_{i, j}^{v}$, we can find the solution of Eqn. (27b) from (37).

Therefore, the following result has been proved.

Theorem 5. The solution of Eqn. (27b) is given by (37) and the vectors $\bar{x}_{i, j}^{h}$ and $\bar{x}_{i, j}^{v}$ are defined by (44) and (40), respectively.

\section{Example}

Consider the descriptor discrete-time linear system (24) with the matrices

$$
\begin{aligned}
E & =\left[\begin{array}{cccc}
0 & 0.5 & 0 & 0 \\
0 & 1 & 0 & 0 \\
0 & 0 & 0 & -1 \\
0 & 0 & 0 & -2
\end{array}\right], \\
A & =\left[\begin{array}{cccc}
0.5 & 0.5 & 0 & 0 \\
0 & 3 & 0 & 0 \\
-1 & -1 & 0 & 2 \\
-2 & 0 & -1 & 5
\end{array}\right], \\
B & =\left[\begin{array}{c}
0 \\
1 \\
-2 \\
4
\end{array}\right] .
\end{aligned}
$$

Premultiplying Eqn. (1) with (45) by the matrix

$$
P=\left[\begin{array}{cccc}
2 & 0 & 0 & 0 \\
-2 & 1 & 0 & 0 \\
0 & 0 & -1 & 0 \\
0 & 0 & -2 & 1
\end{array}\right]
$$

of the elementary row operations $L[1 \times 2], L[2+1 \times(-1)]$, $L[3 \times(-1)], L[4+3 \times 2]$ and introducing the new state vector (26) with

$$
Q=\left[\begin{array}{llll}
0 & 1 & 0 & 0 \\
1 & 0 & 0 & 0 \\
0 & 0 & 0 & 1 \\
0 & 0 & 1 & 0
\end{array}\right]
$$

we obtain Eqn. (27a) with

$$
\begin{gathered}
{\left[\begin{array}{cc}
E_{h} & 0 \\
0 & E_{v}
\end{array}\right]=P E Q^{-1}=\left[\begin{array}{cccc}
1 & 0 & 0 & 0 \\
0 & 0 & 0 & 0 \\
0 & 0 & 1 & 0 \\
0 & 0 & 0 & 0
\end{array}\right],} \\
{\left[\begin{array}{cc}
A_{11} & 0 \\
A_{21} & A_{22}
\end{array}\right]=P A Q^{-1}=\left[\begin{array}{cccc}
1 & 1 & 0 & 0 \\
2 & -1 & 0 & 0 \\
1 & 1 & -2 & 0 \\
2 & 0 & 1 & -1
\end{array}\right],} \\
{\left[\begin{array}{c}
B_{1} \\
B_{2}
\end{array}\right]=P B=\left[\begin{array}{l}
0 \\
1 \\
2 \\
8
\end{array}\right] .}
\end{gathered}
$$

Using the procedure presented for Case 1, we obtain what follows.

For $c_{1}=0$, from (31b) we have

$$
\begin{aligned}
& \bar{E}_{h}=\left[-A_{11}\right]^{-1} E_{h}=\frac{1}{3}\left[\begin{array}{ll}
-1 & 0 \\
-2 & 0
\end{array}\right], \\
& \bar{A}_{11}=\left[-A_{11}\right]^{-1} A_{11}=\left[\begin{array}{cc}
-1 & 0 \\
0 & -1
\end{array}\right], \\
& \bar{B}_{1}=\left[-A_{11}\right]^{-1} B_{1}=\frac{1}{3}\left[\begin{array}{c}
-1 \\
1
\end{array}\right] .
\end{aligned}
$$

Taking into account that

$$
\bar{E}_{h}=V W, \quad V=\frac{1}{3}\left[\begin{array}{l}
-1 \\
-2
\end{array}\right], \quad W=\left[\begin{array}{ll}
1 & 0
\end{array}\right]
$$

and using Procedure A1 from Appendix, we obtain

$$
\begin{aligned}
\bar{E}_{h}^{D}= & V\left[W \bar{E}_{h} V\right]^{-1} W \\
= & \frac{1}{3}\left[\begin{array}{l}
-1 \\
-2
\end{array}\right]\left[\frac{1}{9}\left[\begin{array}{ll}
1 & 0
\end{array}\right]\left[\begin{array}{ll}
-1 & 0 \\
-2 & 0
\end{array}\right]\left[\begin{array}{l}
-1 \\
-2
\end{array}\right]\right]^{-1} \\
& \times\left[\begin{array}{ll}
1 & 0
\end{array}\right] \\
= & {\left[\begin{array}{ll}
-3 & 0 \\
-6 & 0
\end{array}\right] }
\end{aligned}
$$

and

$$
\bar{A}_{11}^{D}=\left[\begin{array}{cc}
-1 & 0 \\
0 & -1
\end{array}\right]
$$


The index $q_{1}$ of the matrix (49) is equal to one. Using (32) and taking into account that

$$
\begin{aligned}
\bar{E}_{h}^{D} \bar{A}_{11} & =\left[\begin{array}{ll}
-3 & 0 \\
-6 & 0
\end{array}\right]\left[\begin{array}{cc}
-1 & 0 \\
0 & -1
\end{array}\right]=\left[\begin{array}{ll}
3 & 0 \\
6 & 0
\end{array}\right], \\
\bar{E}_{h}^{D} \bar{E}_{h} & =\left[\begin{array}{ll}
-3 & 0 \\
-6 & 0
\end{array}\right] \frac{1}{3}\left[\begin{array}{ll}
-1 & 0 \\
-2 & 0
\end{array}\right]=\left[\begin{array}{ll}
1 & 0 \\
2 & 0
\end{array}\right],
\end{aligned}
$$

we obtain

$$
\begin{aligned}
\bar{x}_{i, j}^{h}= & \left(\bar{E}_{h}^{D} \bar{A}_{11}\right)^{i} \bar{E}_{h}^{D} \bar{E}_{h} v_{1}(j) \\
& +\sum_{k=0}^{i-1} \bar{E}_{h}^{D}\left(\bar{E}_{h}^{D} \bar{A}_{11}\right)^{i-k-1} \bar{B}_{1} u_{k, j} \\
& +\left(\bar{E}_{h} \bar{E}_{h}^{D}-I_{n_{1}}\right) \bar{A}_{11}^{D} \bar{B}_{1} u_{i, j} \\
= & {\left[\begin{array}{cc}
3 & 0 \\
6 & 0
\end{array}\right]^{i}\left[\begin{array}{cc}
1 & 0 \\
2 & 0
\end{array}\right] v_{1}(j) } \\
& +\sum_{k=0}^{i-1}\left[\begin{array}{cc}
-3 & 0 \\
-6 & 0
\end{array}\right]\left[\begin{array}{cc}
3 & 0 \\
6 & 0
\end{array}\right]^{i-k-1} \frac{1}{3}\left[\begin{array}{c}
-1 \\
1
\end{array}\right] u_{k, j} \\
& +\left[\begin{array}{cc}
0 & 0 \\
2 & -1
\end{array}\right]\left[\begin{array}{cc}
-1 & 0 \\
0 & -1
\end{array}\right] \frac{1}{3}\left[\begin{array}{c}
-1 \\
1
\end{array}\right] u_{i, j} \\
= & {\left[\begin{array}{cc}
3^{i} & 0 \\
2(3)^{i} & 0
\end{array}\right] v_{1}(j) } \\
& +\sum_{k=0}^{i-1}\left[\begin{array}{cc}
3^{i-k-1} \\
2(3)^{i-k-1}+1
\end{array}\right] u_{k, j}+\left[\begin{array}{c}
0 \\
1
\end{array}\right] u_{i, j},
\end{aligned}
$$

where $v_{1}(j)$ is an arbitrary function of $j$.

Substituting (54) into Eqn. (28b), we obtain (31a) with

$$
\begin{aligned}
E_{v} & =\left[\begin{array}{ll}
1 & 0 \\
0 & 0
\end{array}\right], \quad A_{22}=\left[\begin{array}{cc}
-2 & 0 \\
1 & -1
\end{array}\right] \\
\hat{u}_{i, j} & =\sum_{k=0}^{i-1}\left[\begin{array}{c}
3^{i-k-1} \\
2(3)^{i-k-1}+1
\end{array}\right] u_{k, j}+\left[\begin{array}{l}
0 \\
1
\end{array}\right] u_{i, j} \\
\hat{w}_{i} & =\left[\begin{array}{cc}
3^{i} & 0 \\
2(3)^{i} & 0
\end{array}\right] v_{1}(j)
\end{aligned}
$$

In this case, we choose $c_{2}=0$ and, using (31), we obtain

$$
\bar{E}_{v} \bar{x}_{i, j+1}^{v}=\bar{A}_{22} \bar{x}_{i, j}^{v}+\bar{B}_{2}\left(\hat{u}_{i, j}+\hat{w}_{i}\right),
$$

where

$$
\begin{aligned}
& \bar{E}_{v}=\left[-A_{22}\right]^{-1} E_{v}=\frac{1}{2}\left[\begin{array}{ll}
1 & 0 \\
1 & 0
\end{array}\right], \\
& \bar{A}_{22}=\left[-A_{22}\right]^{-1} A_{22}=\left[\begin{array}{cc}
-1 & 0 \\
0 & -1
\end{array}\right], \\
& \bar{B}_{2}=\left[-A_{22}\right]^{-1}=\frac{1}{2}\left[\begin{array}{ll}
1 & 0 \\
1 & 2
\end{array}\right] .
\end{aligned}
$$

Taking into account that

$$
\bar{E}_{v}=V W, \quad V=\frac{1}{2}\left[\begin{array}{l}
1 \\
1
\end{array}\right], \quad W=\left[\begin{array}{ll}
1 & 0
\end{array}\right]
$$

and using Procedure A1 from Appendix, we obtain

$$
\begin{aligned}
\bar{E}_{v}^{D} & =V\left[W \bar{E}_{v} V\right]^{-1} W \\
& =\frac{1}{2}\left[\begin{array}{l}
1 \\
1
\end{array}\right]\left[\frac{1}{4}\left[\begin{array}{ll}
1 & 0
\end{array}\right]\left[\begin{array}{ll}
1 & 0 \\
1 & 0
\end{array}\right]\left[\begin{array}{l}
1 \\
1
\end{array}\right]\right]^{-1}\left[\begin{array}{ll}
1 & 0
\end{array}\right] \\
& =\left[\begin{array}{ll}
2 & 0 \\
2 & 0
\end{array}\right]
\end{aligned}
$$

and

$$
\bar{A}_{22}^{D}=\left[\begin{array}{cc}
-1 & 0 \\
0 & -1
\end{array}\right] .
$$

Using (58), 59) and (36), we obtain

$$
\begin{aligned}
\bar{x}_{i, j}^{v}= & {\left[\begin{array}{ll}
-2 & 0 \\
-2 & 0
\end{array}\right]^{j} v_{2}(i) } \\
& +\sum_{l=0}^{j-1}\left[\begin{array}{ll}
-2 & 0 \\
-2 & 0
\end{array}\right]^{j-l-1}\left(\hat{u}_{i, l}+\hat{w}_{i}\right) \\
& +\left[\begin{array}{ll}
0 & 0 \\
0 & 1
\end{array}\right]\left(\hat{u}_{i, l}+\hat{w}_{i}\right),
\end{aligned}
$$

where $v_{2}(i)$ is an arbitrary function of $i, \bar{x}_{i, j}^{h 1}$ and $\bar{x}_{i, j}^{h 2}$ are the components of $\bar{x}_{i, j}^{h}$ given by (54).

Knowing the vectors (54), (60) and the matrix (47), we can find the solution of Eqn. (1) with (45) from (37).

\section{Concluding remarks}

A method of analysis for a class of descriptor 2D discrete-time linear systems described by the Roesser model with a regular pencil has been proposed. The method is based on transformation of the descriptor Roesser model (11) to the form (27) with the use of elementary row and column operations. To find a solution to Eqn. (27), a method based on application of the Drazin inverse has been proposed (Theorems 4 and 5). The method has been illustrated with a numerical example. It can be extended to fractional and fractional positive descriptor linear systems described by Roesser models with regular pencils. An extension of the method to 2D descriptor linear systems described by the Fornasini-Marchesini and Kurek models constitutes an open problem.

\section{Acknowledgment}

This work was supported by the Białystok University of Technology under the grant no. S/WE/1/11. 


\section{References}

Bru, R., Coll, C., Romero-Vivo, S. and Sanchez, E. (2003). Some problems about structural properties of positive descriptor systems, in L. Benvenuti, A. de Santis and L. Farina (Eds.), Positive Systems, Lecture Notes in Control and Information Sciences, Vol. 294, Springer, Berlin, pp. 233-240.

Bru, R., Coll, C. and Sanchez, E. (2000). About positively discrete-time singular systems, in M.E. Mastorakis (Ed.), System and Control: Theory and Applications, World Scientific and Engineering Society, Athens, pp. 44-48.

Bru, R., Coll, C. and Sanchez, E. (2002). Structural properties of positive linear time-invariant difference-algebraic equations, Linear Algebra and Its Applications 349: 1-10.

Busłowicz, M. (2014). Controllability, reachability and minimum energy control of fractional discrete-time linear systems with multiple delays in state, Bulletin of the Polish Academy of Sciences: Technical Sciences 62(2): 233-239.

Campbell, S.L., Meyer, C.D. and Rose, N.J., (1976). Applications of the Drazin inverse to linear systems of differential equations with singular constant coefficients, SIAM Journal on Applied Mathematics 31(3): 411-425.

Dai, L. (1989). Singular Control Systems, Lectures Notes in Control and Information Sciences, Springer-Verlag, Berlin.

Dodig, M. and Stosic, M. (2009). Singular systems state feedbacks problems, Linear Algebra and Its Applications 431(8): 1267-1292.

Duan, G.-R. (2010). Analysis and Design of Descriptor Linear Systems, Springer, New York, NY.

Fahmy, M.M, and O'Reill, J. (1989). Matrix pencil of closed-loop descriptor systems: Infinite-eigenvalues assignment, International Journal of Control 49(4): 1421-1431.

Gantmacher, F.R. (1960). The Theory of Matrices, Chelsea Publishing Co., New York, NY.

Kaczorek, T. (1992). Linear Control Systems, Vol. 1, Research Studies Press J. Wiley, New York, NY.

Kaczorek, T. (2002). Positive $1 D$ and $2 D$ Systems, Springer-Verlag, London.

Kaczorek, T. (2004). Infinite eigenvalue assignment by an output feedback for singular systems, International Journal of Applied Mathematics and Computer Science 14(1): 19-23.

Kaczorek, T. (2010). Positive linear systems with different fractional orders, Bulletin of the Polish Academy of Sciences: Technical Sciences 58(3): 453-458.

Kaczorek, T. (2011a). Checking of the positivity of descriptor linear systems by the use of the shuffle algorithm, Archives of Control Sciences 21(3): 287-298.

Kaczorek, T. (2011b). Reduction and decomposition of singular fractional discrete-time linear systems, Acta Mechanica et Automatica 5(4): 62-66.

Kaczorek T. (2011c). Singular fractional discrete-time linear systems, Control and Cybernetics 40(3): 753-761.

Kaczorek, T. (2011d). Selected Problems of Fractional Systems Theory, Springer-Verlag, Berlin.
Kaczorek, T. (2013). Application of the Drazin inverse to analysis of descriptor fractional discrete-time linear systems with regular pencils, International Journal of Applied Mathematics and Computer Science 23(1): 29-33, DOI: 10.2478/amcs-2013-0003.

Kaczorek, T. (2014a). Drazin inverse matrix method for fractional descriptor continuous-time linear systems, Bulletin of the Polish Academy of Sciences: Technical Sciences 62(3): 409-412.

Kaczorek, T. (2014b). Minimum energy control of descriptor positive discrete-time linear systems, COMPEL 33(3): $1-14$.

Kaczorek, T. (2014c). Minimum energy control of positive fractional descriptor continuous-time linear systems, IET Control Theory and Applications 8(4): 219-225.

Kucera, V. and Zagalak, P. (1988). Fundamental theorem of state feedback for singular systems, Automatica 24(5): 653-658.

Van Dooren, P. (1979). The computation of Kronecker's canonical form of a singular pencil, Linear Algebra and Its Applications 27: 103-140.

Virnik, E. (2008). Stability analysis of positive descriptor systems, Linear Algebra and Its Applications 429(10): 2640-2659.

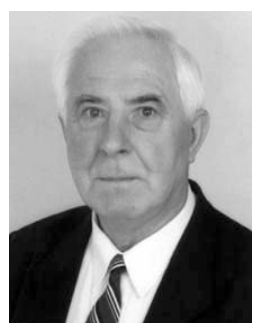

Tadeusz Kaczorek received the M.Sc., Ph.D. and D.Sc. degrees in electrical engineering from the Warsaw University of Technology in 1956, 1962 and 1964, respectively. In the years 1968-69 he was the dean of the Electrical Engineering Faculty, and in the period of 1970-73 he was a deputy rector of the Warsaw University of Technology. In 1971 he became a professor and in 1974 a full professor at the same university. Since 2003 he has been a professor at the Białystok University of Technology. In 1986 he was elected a corresponding member and in 1996 a full member of the Polish Academy of Sciences. In the years 1988-1991 he was the director of the Research Centre of the Polish Academy of Sciences in Rome. In 2004 he was elected an honorary member of the Hungarian Academy of Sciences. He was granted honorary doctorates by 13 universities. His research interests cover systems theory, especially singular multidimensional systems, positive multidimensional systems, singular positive 1D and 2D systems, as well as positive fractional $1 \mathrm{D}$ and $2 \mathrm{D}$ systems. He initiated research in the field of singular $2 \mathrm{D}$, positive $2 \mathrm{D}$ and positive fractional linear systems. He published 28 books ( 8 in English) and over 1000 scientific papers. He also supervised $69 \mathrm{Ph} . \mathrm{D}$. theses. He is the editor-in-chief of the Bulletin of the Polish Academy of Sciences: Technical Sciences and a member of editorial boards of ten international journals.

\section{Appendix}

\section{Procedure for computation of Drazin inverse matrices}

To compute the Drazin inverse $\bar{E}^{D}$ of the matrix $\bar{E} \in \mathbb{R}^{n \times n}$ defined by (3b), the following procedure is recommended. 


\section{Procedure A1.}

Step 1. Find a pair of matrices $V \in \mathbb{R}^{n \times r}, W \in \mathbb{R}^{r \times n}$ such that

$$
\bar{E}=V W, \quad \operatorname{rank} V=\operatorname{rank} W=\operatorname{rank} \bar{E}=r .
$$

As the $r$ columns (rows) of the matrix $V(W)$, the $r$ linearly independent columns (rows) of the matrix $\bar{E}$ can be chosen.

Step 2. Compute the nonsingular matrix

$$
W \bar{E} V \in \mathbb{R}^{r \times r} .
$$

Step 3. The desired Drazin inverse matrix is given by

$$
\bar{E}^{D}=V[W \bar{E} V]^{-1} W .
$$

Proof. It will be shown that the matrix (A3) satisfies the three conditions (5) of Definition 2. Taking into account that $\operatorname{det} W V \neq 0$ and (A1), we obtain

$$
[W \bar{E} V]^{-1}=[W V W V]^{-1}=[W V]^{-1}[W V]^{-1} .
$$

Using (5), (A1) and (A4), we obtain

$$
\begin{aligned}
\bar{E} \bar{E}^{D} & =V W V[W \bar{E} V]^{-1} W \\
& =V W V[W V]^{-1}[W V]^{-1} W \\
& =V[W V]^{-1} W
\end{aligned}
$$

and

$$
\begin{aligned}
\bar{E}^{D} \bar{E} & =V[W \bar{E} V]^{-1} W V W \\
& =V[W V]^{-1}[W V]^{-1} W V W \\
& =V[W V]^{-1} W .
\end{aligned}
$$

Therefore, the condition (5) is satisfied.
To check the condition (5), we compute

$$
\begin{aligned}
\bar{E}^{D} \bar{E} \bar{E}^{D} & =V[W \bar{E} V]^{-1} W V W V[W \bar{E} V]^{-1} W \\
& =V[W V W V]^{-1} W V W V[W \bar{E} V]^{-1} W \\
& =V[W \bar{E} V]^{-1} W=\bar{E}^{D} .
\end{aligned}
$$

Therefore, the condition (5) is also satisfied.

Using (5), (A1), (A3) and (A4), we obtain

$$
\begin{aligned}
\bar{E}^{D} \bar{E}^{q+1} & =V[W \bar{E} V]^{-1} W(V W)^{q+1} \\
& =V[W V]^{-1}[W V]^{-1} W V W(V W)^{q} \\
& =V[W V]^{-1} W(V W)^{q} \\
& =V W(V W)^{q-1} \\
& =(V W)^{q}=\bar{E}^{q},
\end{aligned}
$$

where $q$ is the index of $\bar{E}$. Therefore, the condition (5) is also satisfied.

Received: 16 May 2014 